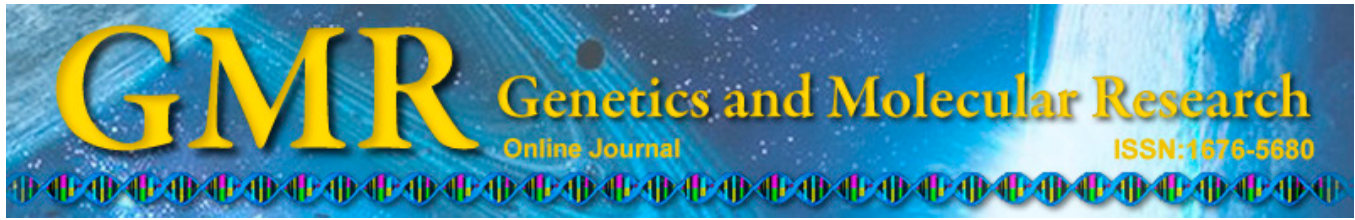

\title{
Analysis of prognostic factors for infantile rotavirus infection
}

\author{
R. Zhou ${ }^{1,2}$, J.L. Xu ${ }^{2}$, D. Wu ${ }^{1}$ and J.L. Tang ${ }^{1}$ \\ ${ }^{1}$ Department of Pediatrics, \\ The First Affiliated Hospital of Anhui Medical University, Hefei, China \\ ${ }^{2}$ Department of Pediatrics, \\ The First Affiliated Hospital of Bengbu Medical College, Bengbu, China \\ Corresponding author: J.L. Tang \\ E-mail: jiulaitang@163.com
}

Genet. Mol. Res. 14 (1): 790-796 (2015)

Received April 16, 2014

Accepted September 7, 2014

Published February 2, 2015

DOI http://dx.doi.org/10.4238/2015.February.2.3

\begin{abstract}
In this study, we investigated the prognostic factors of infantile rotavirus (RV) infection. A total of 102 infants with RV enteritis were divided into 2 groups according to the standards of improvement and cure at the time of discharge from the hospital: improvement group $(\mathrm{N}=58 ; 47$ males and 11 females with an average age of $15.19 \pm 5.03$ months) and the cure group ( $\mathrm{N}=44 ; 34$ males and 10 females with an average age of $10.02 \pm 4.92$ months). Both groups were phlebotomized for the detection of serum glutamic oxaloacetic aminotransferase, creatine kinase-MB, and lactate dehydrogenase. Prognostic factors and clinical data were analyzed by univariate and multivariate logistic analysis. Among the 102 cases of RV infection, 58 were cured and 44 were improved. Univariate analysis showed that the 2 groups were significantly different in age, feeding pattern, concentrations of serum glutamic oxaloacetic aminotransferase, creatine kinase-MB, and lactate dehydrogenase, and central nervous system damage. Logistic regression analysis showed that age, feeding, and central nervous system damage were significant independent prognostic factors for RV enteritis $(\mathrm{P}<0.05)$. There were no statistical differences in gender,
\end{abstract}


course of disease, and respiratory infection $(\mathrm{P}<0.05)$. Both myocardial and hepatic damages presented a temporary feature in the infants and had no significant influence on prognosis. Age, feeding pattern, and central nervous system damage are significant independent prognostic factors for RV infection. These factors should be carefully considered in clinical practice.

Key words: Diarrhea; Factor; Prognosis; Rotavirus

\section{INTRODUCTION}

Rotavirus (RV) is the most common pathogen causing severe diarrhea in children under 5 years old worldwide. Nearly all children under 5 years have experienced gastroenteritis caused by RV infection, both in developed and developing countries. In addition to enteral infection, RV can cause parenteral damage (Chiappini et al., 2005; Ramani et al., 2010). RV infection has a sudden onset and is often accompanied by symptoms such as fever, vomiting, and diarrhea. Severe infection can result in life-threatening dehydration and electrolyte disturbances. In developing countries, infantile diarrhea is a primary cause for deaths, ranking 2 nd after pneumonia (Parashar et al., 2006; Black et al., 2010). However, because of a variety of RV subtypes and lack of satisfactory cross protection, an effective prevention and cure method for RV infection has not been developed (Desselberger and Huppertz, 2011).

To explore the prognostic factors for infantile RV infection, we retrospectively analyzed the prognoses, general conditions, and parenteral damages of 102 infants with RV enteritis.

\section{MATERIAL AND METHODS}

\section{Subjects}

A total of 102 children presenting water- or egg drop soup-like stools accompanied by clinical symptoms such as vomiting and fever received treatment at the First Affiliated Hospital of Anhui Medical University between July 2007 and July 2010. All patients had complete data. This study was conducted in accordance with the Declaration of Helsinki and approved by the Ethics Committee of the First Affiliated Hospital of Anhui Medical University. Written informed consent was obtained from all participants' guardians.

The first stool sample after hospitalization was collected from each child for RV antigen detection using the gold-labeled method. All children were confirmed to have RV infection and met the diagnostic criteria of RV enteritis (Medici et al., 2011). They were all confirmed to have no histories of congenital heart disease, myocarditis, toxic encephalopathy, hepatorenal diseases, and various muscle diseases. They had no medical history of apparent respiratory or alimentary infection within 1 month. Cure criteria included a noticeable improvement in stool characteristics within 3 days after treatment, at least 2 times of defecation reduced daily, no parenteral symptoms, and a negative result within 7 days according to clinical stool RV antigen detection (Fang, 2011). Improvement criteria included a marked reduction in the number of defecations within 3 days after treatment compared with before treatment, an improvement in stool characteristics, and disappearance or noticeable alleviation of other clinical symptoms (Fang, 2011). Based on these criteria, the patients were divided into cure and improvement 
groups ( $\mathrm{N}=58$ and 44 , respectively). The cure group included 47 males and 11 females with an average age of $15.19 \pm 5.03$ months. The improvement group included 34 males and 10 females with an average age of $10.02 \pm 4.92$ months.

\section{Detection methods and judgment criteria}

Fresh stools were collected from both groups immediately after hospitalization. RV antigen detection was performed using the gold labeled method (the kit was supplied by Beijing Wantai Biological Pharmacy, China). Venous blood (approximately $2 \mathrm{~mL}$ ) was extracted on an empty stomach in the morning. Serum glutamic oxaloacetic aminotransferase (SCOT), creatine kinase-MB (CK-MB), and lactate dehydrogenase (LDH) were continuously determined using an AU5400 automatic biochemistry analyzer (Olympus, Tokyo, Japan). The judgment criteria of parenteral damages were as follows: 1) hepatic damage: SCOT $>40 \mathrm{U} / \mathrm{L} ; 2$ ) myocardial damage: $\mathrm{CK}-\mathrm{MB}>25 \mathrm{U} / \mathrm{L}$ and $\mathrm{LDH}>215 \mathrm{U} / \mathrm{L} ; 3)$ respiratory tract infection: cough, nasal discharge, thickened bilateral pulmonary respiratory sounds, and increased bilateral pulmonary markings according to chest radiography; and 4) central nervous system (CNS) damage: altered consciousness, convulsions, intracranial hypertension, or changes in cerebrospinal fluid.

\section{Statistical analysis}

Data were analyzed using the SPSS13.0 software (SPSS, Inc., Chicago, IL, USA). Measurement data are reported as means \pm standard error (means $\pm \mathrm{SE}$ ) and tested using $t$ test. The $\chi^{2}$ test was used to compare rates. Logistic regression analysis was performed for multivariate analysis with odd ratios and their $95 \%$ confidence intervals $(\mathrm{CI})$ for relative risks. $\mathrm{P}<$ 0.05 was considered to be statistically significant.

\section{General data and univariate analysis}

Among the 102 patients, 58 recovered and 44 improved. Univariate analyses of 9 indices were performed between groups. The ages, feeding patterns, SCOT concentrations, CK-MB concentrations, LDH concentrations, and the presence or absence of CNS damage of the 2 groups showed significant differences $(\mathrm{P}<0.05)$, whereas gender, courses of disease, and the presence or absence of respiratory infection did not $(\mathrm{P}>0.05)$. The results are summarized in Tables 1 and 2.

\begin{tabular}{|c|c|c|c|c|c|c|c|}
\hline \multirow[t]{2}{*}{ Group } & \multirow[t]{2}{*}{ Case number } & \multicolumn{2}{|c|}{ Gender } & \multirow[t]{2}{*}{ Age (months) } & \multirow[t]{2}{*}{ Course of disease (days) } & \multicolumn{2}{|c|}{ Feeding pattern } \\
\hline & & Male & Female & & & Breast & Artificial \\
\hline Cure & 58 & 47 & 11 & $15.19 \pm 5.031$ & $9.33 \pm 4.85$ & 48 & 10 \\
\hline Improvement & 44 & 34 & 10 & $10.02 \pm 4.92$ & $15.3 \pm 6.85$ & 29 & 15 \\
\hline t or $\chi$ value & & 0.217 & & 8.50 & 6.70 & 3.839 & \\
\hline P value & & 0.642 & & 0.01 & 0.037 & $<0.05$ & \\
\hline
\end{tabular}


Table 2. Parenteral damages in both groups.

\begin{tabular}{|c|c|c|c|c|c|c|c|}
\hline \multirow[t]{2}{*}{ Group } & \multirow{2}{*}{$\begin{array}{c}\text { Hepatic damage }(\mathrm{U} / \mathrm{L}) \\
\text { SCOT }\end{array}$} & \multicolumn{2}{|c|}{ Myocardial damage (U/L) } & \multicolumn{2}{|c|}{ Respiratory infection } & \multicolumn{2}{|c|}{ CNS damage } \\
\hline & & CK-MB & $\mathrm{LDH}$ & Yes & No & Yes & No \\
\hline Cure & $52.74 \pm 18.02$ & $36.63 \pm 9.13$ & $230.30 \pm 48.78$ & 30 & 28 & 4 & 54 \\
\hline Improvement & $90.19 \pm 22.06$ & $70.63 \pm 18.62$ & $360.55 \pm 36.24$ & 22 & 22 & 15 & 29 \\
\hline t or $\chi$ value & -40.41 & -27.88 & -7.65 & 0.546 & 12.207 & \multirow{2}{*}{\multicolumn{2}{|c|}{0.001}} \\
\hline P value & 0.001 & 0.001 & 0.002 & \multicolumn{2}{|c|}{0.460} & & \\
\hline
\end{tabular}

\section{Multivariate analysis}

Logistic regression analysis was performed for all univariate indices using the SPSS13.0 software. The binary categorical variables were 0 for clinical cure and 1 for improvement. The analysis showed that age, feeding pattern, and CNS damage were 3 significant independent risk factors for the prognosis of infantile RV enteritis. The results are summarized in Table 3.

Table 3. Logistic regression analysis of prognosis associated risk factors of infantile RV enteritis.

\begin{tabular}{lcccc}
\hline Variable & Partial regression coefficient & Standard error of partial regression coefficient & P value & Odds ratio (95\%CI) \\
\hline Age & 2.526 & 0.659 & 0.021 & $9.602(1.265-16.472)$ \\
Feeding pattern & 3.352 & 0.818 & 0.001 & $28.533(5.749-141.802)$ \\
CNS damage & 2.002 & 0.518 & 0.009 & $19.339(5.757-14.962)$ \\
\hline
\end{tabular}

\section{DISCUSSION}

RV enteritis is a commonly and frequently encountered disease in infants. RV infection-related diarrhea results in at least 600,000 deaths annually in developing countries. However, to date, no effective drugs for RV infection have been developed (Parashar et al., 1998; Grandy et al., 2010). There are 7 types of RV. Among them, group A includes the most common viruses that primarily affect infants. RV can lead to digestive system symptoms such as vomiting and diarrhea, as well as the involvement of other systems such as myocardial and hepatorenal damages (Ramani et al., 2010). In this study, all 102 infants with RV enteritis showed varying severity of myocardial and hepatorenal damages or respiratory system infection and CNS damage, which was consistent with previously reported observations (Liu et al., 2009; Fujita et al., 2010).

The factors associated with patients' prognoses such as the general conditions and parenteral damages were analyzed. Univariate analysis showed that the cure and improvement groups displayed significant differences between the ages, feeding patterns, SCOT concentrations, CK-MB concentrations, and LDH concentrations, as well as the presence or absence of CNS damage. This indicates that the prognosis of RV infection is correlated with onset age, feeding pattern, and hepatic, myocardial, and CNS damages. RV infection primarily occurs in infants between 6 months and 2 years of age (Macartney et al., 2011). In this study, the average age of the improvement group was lower than that of the cure group. This may be attributable to the immaturity of intestinal immune functions because of their younger age or a poor virusadhering and -neutralizing capacity resulting from lower immunoglobulin A (IgA) level (Di Niro et al., 2010). Feeding pattern also influences prognosis. In this study, the proportion of infants fed breast milk in the cure group was higher than that in the improvement group. Currently, the role of breast feeding in preventing infantile RV infection has attracted increased at- 
tention. Different feeding patterns have varying impacts on the occurrence of RV diarrhea, the severity of RV infection, and the treatment effect on RV infection (Deng et al., 2007). Breastfeeding prevents and reduces the incidence of infantile RV infection; additionally, it eases the severity of infantile dehydration and diarrhea and shortens average hospital days compared with artificial feeding (Gu and Shen, 2010). These effects of breast-feeding are thought to be associated with the action of secretory $\operatorname{IgA}(\operatorname{sg} A$ ) in breast milk. However, recent studies have shown that large amounts of oligosaccharide and complex carbohydrates in breast milk, particularly lactadherin (a type of lipophilic glycoprotein on fat globule membrane surfaces), also play similar preventive roles (Shi and Gilbert, 2003). Lactadherin can evoke a series of vital movements by binding to the $\alpha \mathrm{V} \beta 3$ and $\alpha \mathrm{V} \beta 5$ integrin receptors in various cells in vivo. It can also regulate the activity of gastrointestinal immune cells, strengthening their anti-RV capacity and thus benefiting the prevention and cure of RV infection (Black and Armstrong, 1998; Newburg et al., 1998; Shahriar et al., 2006). Furthermore, in this study, the concentrations of SCOT, CK-MB, and LDH in the cure group were lower than those in the improvement group. This may be related to the severity of hepatic and myocardial damages and pathological conditions at the time of RV infection. Severe hepatic cell damage may provide a satisfactory foundation for virus replication (Liu et al., 2009; Fujita et al., 2010). However, a previous study showed that the younger the age of onset, the higher the risk of hepatic damage; hepatic damage is age-correlated rather than pathological condition-correlated (Fang, 2011). Another similar study showed that a younger age is related to higher incidence rates of hepatic and myocardial damages (Black and Armstrong, 1998). The increased activity of serum myocardial enzymes are closely correlated with pathological conditions; the more severe the degree of dehydration, the more significantly the activity of myocardial enzymes will increase (Liu et al., 2009; Fujita et al., 2010). A potential explanation for this observation may be correlated with the barrier function of the infantile intestinal mucosa: SIgA insufficiency facilitates the access of the virus through the barrier into the blood circulation. Although increases in myocardial enzymes may be correlated with fever, dehydration, and pachyemia, whether RV and its toxins cause direct myocardial damage remains unknown. Moreover, in this study, some infants presented convulsions, for which aseptic meningitis cannot be excluded (Tang et al., 2010). The existence of RV in the cerebrospinal fluid in infants with RV enteritis complicated by meningitis suggests that RV can lead to viremia (Medici et al., 2011), which can lead to CNS damage (Kawano et al., 2007). However, it has been suggested that both RV-caused viremia and brain damage lack a reliable theoretical basis (Fang, 2011). This study shows that CNS damage may be a prognostic factor for RV. Nevertheless, this result must be further explored.

Because univariate analysis could not completely exclude the possible impacts of causal factors and confounding factors in this study, multivariate logistic regression analysis was performed. The results showed that age, feeding pattern, and CNS damage were significant independent risk factors for the prognosis of infantile RV enteritis. Although RV enteritis is often complicated by hepatic functional damage (Rauschenfels et al., 2009; Kawashima et al., 2012; Akelma et al., 2013), multivariate logistic analysis revealed no correlation between hepatic functional damage and the prognosis of RV enteritis. This may be because myocardial and hepatic damages are temporary rather than irreversible in most infants with RV enteritis. Therefore, both groups can recover after active symptomatic treatment and liver-protecting and myocardium-nourishing treatment. However, to avoid the aggravation of pathological conditions or even sudden cardiac death, electrocardiography and myocardial enzymogram examination should be performed to determine whether RV enteritis is complicated by he- 
patic and myocardial damages. Furthermore, breast-feeding should be advocated and actively popularized.

\title{
Conflicts of interest
}

\author{
The authors declare no conflict of interest.
}

\section{REFERENCES}

Akelma AZ, Kütükoğlu I, Köksal T, Cizmeci MN, et al. (2013). Serum transaminase elevation in children with rotavirus gastroenteritis: seven years' experience. J. Scand J. Infect. Dis. 45: 362-367.

Black ME and Armstrong D (1998). Human-milk lactadherin in protection against rotavirus. Lancet 351: 1815-1816.

Black RE, Cousens S, Johnson HL, Lawn JE, et al. (2010). Global, regional, and national causes of child mortality in 2008: a systematic analysis. Lancet 375: 1969-1987.

Chiappini E, Azzari C, Moriondo M, Galli L, et al. (2005). Viraemia is a common finding in immunocompetent children with rotavirus infection. J. Med. Virol. 76: 265-267.

Deng L, Jia LY, Zhao HX, Zhang Y, et al. (2007). Clinical manifestations of 140 infants with rotavirus enteritis. J. Clin. Pediatrics 25: 295-297.

Desselberger U and Huppertz HI (2011). Immune responses to rotavirus infection and vaccination and associated correlates of protection. J. Infect. Dis. 203: 188-195.

Di Niro R, Mesin L, Raki M, Zheng NY, et al. (2010). Rapid generation of rotavirus-specific human monoclonal antibodies from small-intestinal mucosa. J. Immunol. 185: 5377-5383.

Fang HS (2011). Diagnosis and treatment of childhood diarrhea disease. J. Applied Clin. Pediatrics 26: 1537-1540.

Fujita Y, Liu B, Kohira R, Fuchigami T, et al. (2010). Rotavirus antigenemia and genomia in children with rotavirus gastroenteritis. J. Infect. Dis. 63: 83-86.

Grandy G, Medina M, Soria R, Terán CG, et al. (2010). Probiotics in the treatment of acute rotavirus diarrhoea. A randomized, double-blind, controlled trial using two different probiotic preparations in Bolivian children. BMC Infect. Dis. 10: 253.

Gu Z and Shen HQ (2010). The effects of breast feeding or bottle feeding on infants with rotavirus diarrhea. J. Clin. Pediatrics. 28: 57-60.

Kawano G, Oshige K, Syutou S, Koteda Y, et al. (2007). Benign infantile convulsions associated with mild gastroenteritis: a retrospective study of 39 cases including virological tests and efficacy of anticonvulsant. J. Brain Dev. 29: 617-622.

Kawashima H, Ishii C, Ioi H, Nishimata S, et al. (2012). Transaminase in rotavirus gastroenteritis. J. Pediatr. Int. 54: 86-88.

Liu B, Fujita Y, Arakawa C, Kohira R, et al. (2009). Detection of rotavirus RNA and antigens in serum and cerebrospinal fluid samples from diarrheic children with seizures. J. Infect. Dis. 62: 279-283.

Macartney KK, Porwal M, Dalton D, Cripps T, et al. (2011). Decline in rotavirus hospitalisations following introduction of Australia's national rotavirus immunisation programme. J. Paediatr. Child Health 47: 266-270.

Medici MC, Abelli LA, Guerra P, Dodi I, et al. (2011). Case report: detection of rotavirus RNA in the cerebrospinal fluid of a child with rotavirus gastroenteritis and meningism. J. Med. Virol. 83: 1637-1640.

Newburg DS, Peterson JA, Ruiz-Palacios GM, Matson DO, et al. (1998). Role of human-milk lactadherin in protection against symptomatic rotavirus infection. Lancet 351: 1160-1164.

Parashar UD, Holman RC, Clarke MJ, Bresee JS, et al. (1998). Hospitalizations associated with rotavirus diarrhea in the United States, 1993 through 1995: surveillance based on the new ICD-9-CM rotavirus-specific diagnostic code. $J$. Infect. Dis. 177: 13-17.

Parashar UD, Gibson CJ, Bresee JS and Glass RI (2006). Rotavirus and severe childhood diarrhea. J. Infect. Dis. 12: 304-306.

Ramani S, Paul A, Saravanabavan A, Menon VK, et al. (2010). Rotavirus antigenemia in Indian children with rotavirus gastroenteritis and asymptomatic infections. Clin. Infect. Dis. 51: 1284-1289.

Rauschenfels S, Krassmann M, Al-Masri AN, Verhagen W, et al. (2009). Incidence of hepatotropic viruses in biliary atresia. J. Eur. J. Pediatr. 168: 469-476.

Shahriar F, Ngeleka M, Gordon JR and Simko E (2006). Identification by mass spectroscopy of F4ac-fimbrial-binding proteins in porcine milk and characterization of lactadherin as an inhibitor of F4ac-positive Escherichia coli attachment to intestinal villi in vitro. Dev. Comp. Immunol. 30: 723-734. 
Shi J and Gilbert GE (2003). Lactadherin inhibits enzyme complexes of blood coagulation by competing for phospholipidbinding sites. Blood 101: 2628-2636.

Tang H, Wang Q and Zheng JL (2010). Clinical analysis of human rotavirus infection combined with parenteral damage in infants and young children. Chin. J. General Practice 8: 196-197. 